\title{
A TRANSPONDER FOR GIGABIT ETHERNET OVER WDM
}

\author{
D.A. Schupke ${ }^{1}$ \\ and S. Weigert ${ }^{1}$ \\ and O. Jahreis ${ }^{2}$ \\ ${ }^{1}$ Munich University of Technology \\ Institute of Communication Networks \\ 80290 Munich, Germany \\ Tel.: +4989 289-23511, Fax: +4989 289-63511 \\ Dominic.Schupke@lkn.ei.tum.de \\ Stefan.Weigert@t-online.de \\ ${ }^{2}$ Optisphere Networks Inc., A Siemens Company \\ Reston, VA, U.S.A. \\ Tel.: +1 703-262-2507 \\ oliver.jahreis@optisphere.com
}

\begin{abstract}
The deployment of Gigabit Ethernet in corporate and ISP networks makes the transmission over WDM networks necessary to provide for the interconnection over metropolitan and wide area WDM networks. As the specifications of the optical signal between Gigabit Ethernet and the WDM-network mismatch, a transponder is needed. We propose an architecture for a Gigabit Ethernet over WDM transponder and report on a transponder prototype implementation. We further show how to use the transponder for Gigabit Ethernet networks over WDM rings.
\end{abstract}

\section{INTRODUCTION}

The interconnection of decentralized corporate networks to form a virtual private network (VPN) gains more and more importance today. At the same time wavelength-division multiplex (WDM) networks are emerging supporting multiple protocol-transparent client links and are therefore best suited to provide for broadband VPNs. One important WDM network architecture is the ring-based metropolitan area network (MAN).

The original version of this chapter was revised: The copyright line was incorrect. This has been corrected. The Erratum to this chapter is available at DOI: 10.1007/978-0-387-35491-0_28 
As currently most corporate local area networks (LAN) are Ethernet based, one efficient method is to interconnect these LANs by Gigabit Ethernet over WDM-links [1], if they are demanding high bandwidth among each other. By this the coexistence of multiple protocols and protocol conversion at the LAN/MAN-edge is avoided.

Gigabit Ethernet has also gained importance as "routed Gigabit Ethernet," where this protocol is used as a point-to-point data link only [2]. Gigabit Ethernet performs no switching and is only present at the ports of an IP router. Thus routed Gigabit Ethernet can form the backbone of an Internet service provider (ISP).

In both cases inexpensive Ethernet equipment can be used, which is popular and known among network users.

Gigabit Ethernet, however, cannot be directly carried over a transport WDM-network. The standard port signal has to be adjusted to the specifications of the WDM-network which is accomplished by a transponder.

In section 2 we propose an architecture for a Gigabit Ethernet transponder and describe a prototype implementation based on this in section 3 . We discuss in section 4 the issue of the transponder location and show in section 5 how the transponder fits in favorite network structures. In section 6 we conclude this paper.

\section{TRANSPONDER ARCHITECTURE}

An optical Gigabit Ethernet connection is operated in full-duplex mode. The $1 \mathrm{Gbit} / \mathrm{s}$ client signal is encoded by a $8 \mathrm{~B} 10 \mathrm{~B}$ line-code to achieve DC balance (which is important for the setting of the receiver's threshold) and to cancel long 0/1-sequences (which is needed for clock recovery at the receiver) [3].

The encoding results in a symbol rate of 1.25 Gbaud. The main function of the transponder is to convert this Gigabit Ethernet signal present in 1000BASE-LX or -SX (as defined in [4]) to a WDM signal. Moreover the transmission power at the sender and the sensitivity at the receiver has to be adjusted.

We propose the basic structure depicted in the block diagram of figure 1 . On the left side is the optical in/output to connect to the end system in normal operation (working).

To achieve network redundancy as described in section 5 the end system signal can be switched by $\mathrm{S} 1$ to a protection in/output. There it is possible to connect to a second end system (optically) or to another transponder (electrically). At S1 the input signal is chosen based on 


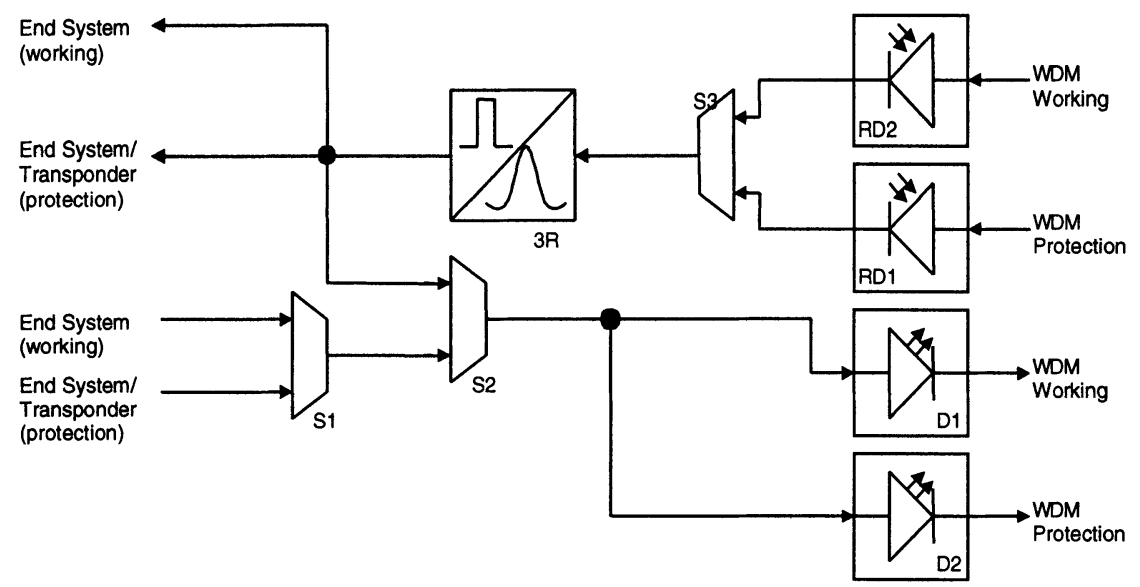

Figure 1: Transponder block diagram

the Loss-of-Signal (LOS) indication of the electrical preamplifier (not shown).

On the right side are working and protection interfaces for the WDM signal to allow for $1+1$ protection on the WDM network. With $1+1$ protection the signal is fed into two laser sources and thus transmitted redundantly. The receiver obtains both signals and chooses with S3 the forward signal based on the received power.

We propose a modular transmit-receive-unit for both working and protection instances. Therefore the protection unit can be omitted if $1+1$ protection is unnecessary. This reduces the cost of the transponder, since expensive laser optic can be saved.

As we do o/e/o-conversion at the transponder, in the receiver part we can easily incorporate electrical 3R-regeneration (resizing, retiming, reshaping) of the signal chosen by S3. This is introduced, since the signal carried over the WDM network can have already low quality. The regenerated signal then goes to the two outputs for the end systems simultaneously.

For fault localization or testing a loopback can be performed at S2. In normal operation S2 switches the input signal to the sending unit(s). In loopback state S2 switches the regenerator signal to the sending unit(s). Thus the WDM signal is received, regenerated and sent back.

To obtain a stable WDM signal of both senders, a separate controler for the lasers is needed, too (not shown). 


\section{PROTOTYPE IMPLEMENTATION}

The transponder prototype was developed based on the structure shown in figure 1. It is further equipped with a controler which is responsible for evaluation of measured data, supervising the hardware and performing some additional functions for Operation and Maintenance (OAM).

The controler can be connected to the Gigabit Ethernet network to use the error database of the equipment. By this the transponder is able to get, e.g., information about the frame error rate which is provided by the simple network management protocol (SNMP). This is important for performance monitoring, because Ethernet does not prove bit error rate monitoring.

The SNMP interface can also be used for the management of the transponder from a remote network management system. Because of the utilization of the Gigabit Ethernet infrastructure no additional management and control links between the transponder and the network management system are necessary.

For radiation protection an automatic laser safety mechanism was also implemented in the transponder. This mechanism works without a signaling system, but is realized on the optical layer.

The selection of the receive paths is made by the measurement of the optical input power

The system measures for each of the two redundant lines the analog optical input power, which is digitized by the controler. If the input power is too low to regenerate the data stream, traffic will be routed over the redundant line and the corresponding laser diode will be switched off. At the far end of the optical link between the two transponders the same mechanism is used. When the bi-directional link is down, the transmitters at each end of the system probe the link periodically with short flashes of light. Only if one of these flashes can be received at the system's far end, the lasers are activated again.

The prototype is realized as a set of boards (euro format). One of these boards (the controler board) contains all the functionality for switching, signal regeneration and measurement. On the controler board the analog signals are switched by 2 to 1 multiplexers. The electrical path between the optical front ends is controled and initialized by the controler of the transponder.

The redundant transmitters and receivers are made as two identical boards, so the transponder can be equipped with either one or two optical WDM-ports. These transmit-receive-units contain the optical front ends (laser and receiver diode) with the transmission stages (an amplifier in 


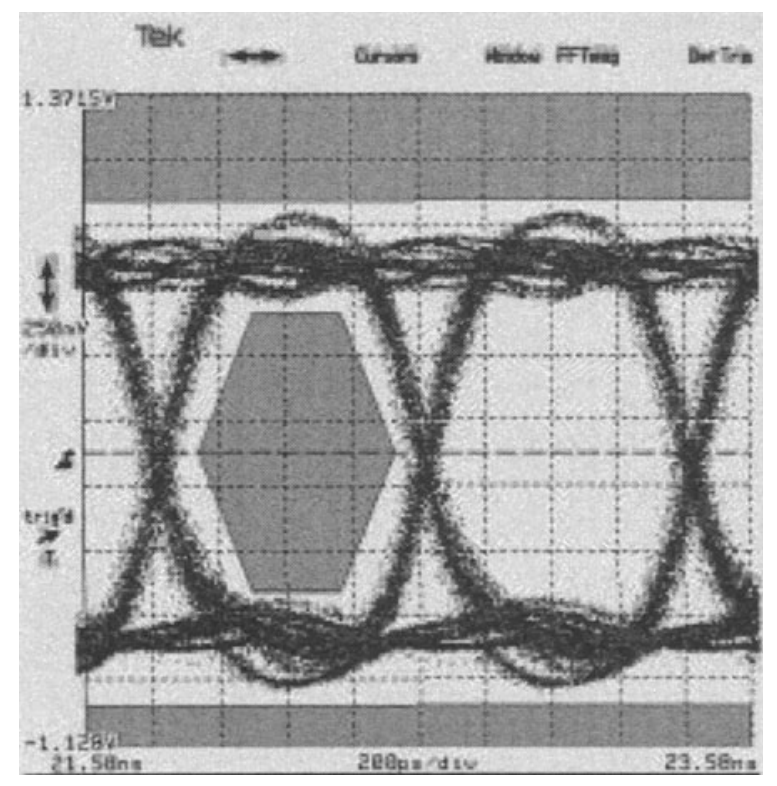

Figure 2: Eye diagram of the received signal

the receive path and a driver in the transmission path). Furthermore each of the transmit-receive-units uses an external laser diode controler in the prototype, in order to control the wavelength of the transmission diode.

The 3R-regenerator was provided as a single chip solution. The regenerator can be switched to different bit rates up to $1.25 \mathrm{Gbit} / \mathrm{s}$. For this reason the transponder can also be used for rates under $1.25 \mathrm{Gbit} / \mathrm{s}$.

The WDM-ports of the prototype maintain a sensitivity of at least $-25 \mathrm{dBm}$ and a maximum output power of $+3 \mathrm{dBm}$. The wavelength divergence is at most $\pm 0.001 \mathrm{~nm}$. The eye diagram of a received and regenerated Gigabit Ethernet signal is depicted in figure 2 (approx. 15$20 \mathrm{~dB}$ attenuation). The signal is in the white area and thus fulfills the requirements of [4].

\section{TRANSPONDER LOCATION}

For the employment in a network we have to determine where to install the transponder appropriately: at the end system location or at the WDM access node.

One decision parameter is whether the network operator of the Gigabit Ethernet equipment or the WDM network operator is responsible for 
operation and management of the transponder. We assume the latter case in the following.

If the transponder is located at the WDM node, the optical power of the WDM channel has to be adjusted for the WDM network only. The WDM operator has an easy access to the transponder and can perform out-band management of the transponder. But using standard Gigabit Ethernet ports for transmission, the access link is restricted to span a short distance $(5 \mathrm{~km})$.

If the transponder is located at the Gigabit Ethernet equipment, we obtain a definite network termination. Since the distance from the end system to the transponder can be short (in-house, some 10 meters), we can also use an inexpensive copper connection (1000BASE-CX or -T) between the two elements. As the optical power of the WDM channel at the WDM access node, however, is dependent on the distance of the access link, more adjustment is necessary and high power levels may be reached. In absence of parallel access links, in-band signaling has to be used for managing the transponder.

Another criterion is the protection of the access link. The transponder can perform this protection only if located at the Gigabit Ethernet equipment. If located at the WDM node, the protection from the end system to the transponder has to be modified.

\section{NETWORK STRUCTURES}

In figure 3 we show a realization of a Gigabit Ethernet line network A-B-C over a WDM ring, e.g. a transparent MAN ring. The line network consists basically of two point-to-point links between A-B and B-C, each requiring transponders at the endpoints. Since node $B$ can fail and a communication between $\mathrm{A}$ and $\mathrm{C}$ should still be possible, $\mathrm{B}$ can be bypassed by the two adjacent transponders. For this the protection in/outputs of the transponders are connected by a bypass link. If node B fails, S1 in both transponders switch to the protection connection.

Figure 4 depicts a star network over a WDM ring, where the nodes A, $\mathrm{C}$ and D are connected to the star node B. Node B has a backup node B' for redundancy. Here the protection in/outputs of the transponders are used to connect the nodes A, C and D to node B' if node B failed.

Usually, Gigabit Ethernet also provides by itself survivability mechanisms like load-balancing, 1+1-protection and reconfiguration by the spanning tree algorithm.

$1+1$-protection requires two and load-balancing requires at least two ports and two connections with transponders between the Gigabit Ethernet end systems. However, to not overprovide redundancy the transpon- 


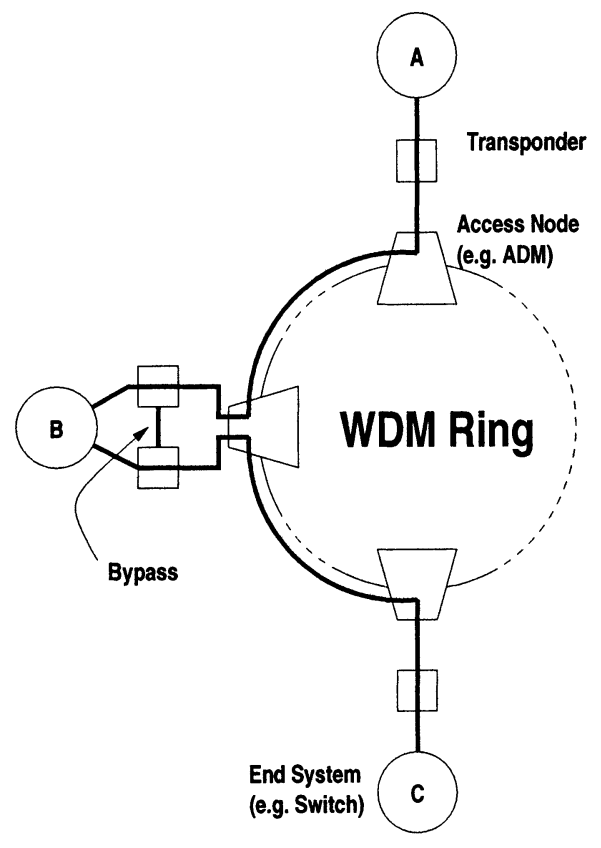

Figure 3: A line network over a WDM ring

ders only need to be equipped with one transmit-receive-unit (working). By this, the link from the Gigabit Ethernet equipment to the WDM ring can be made redundant, too. For instance, two disjoint access links and WDM nodes can be used (dual homing).

\section{CONCLUSIONS}

We presented an architecture for a Gigabit Ethernet over WDM transponder with modular transmit-receive-units for working and protection. The transponder also does 3R-regeneration, automatic laser safety switching and is manageable over Gigabit Ethernet. The functionality of the transponder was demonstrated by a prototype. We have further shown how to locate the transponder (geographically and administratively). We presented possible configurations of Gigabit Ethernet line and star networks over WDM rings.

From the technical point of view the bandwidth can be increased easily to $2.5 \mathrm{Gbit} / \mathrm{s}$. This becomes an issue for transmissions of future 10 Gigabit Ethernet rates which are composed of four $2.5 \mathrm{Gbit} / \mathrm{s}$ signals. 


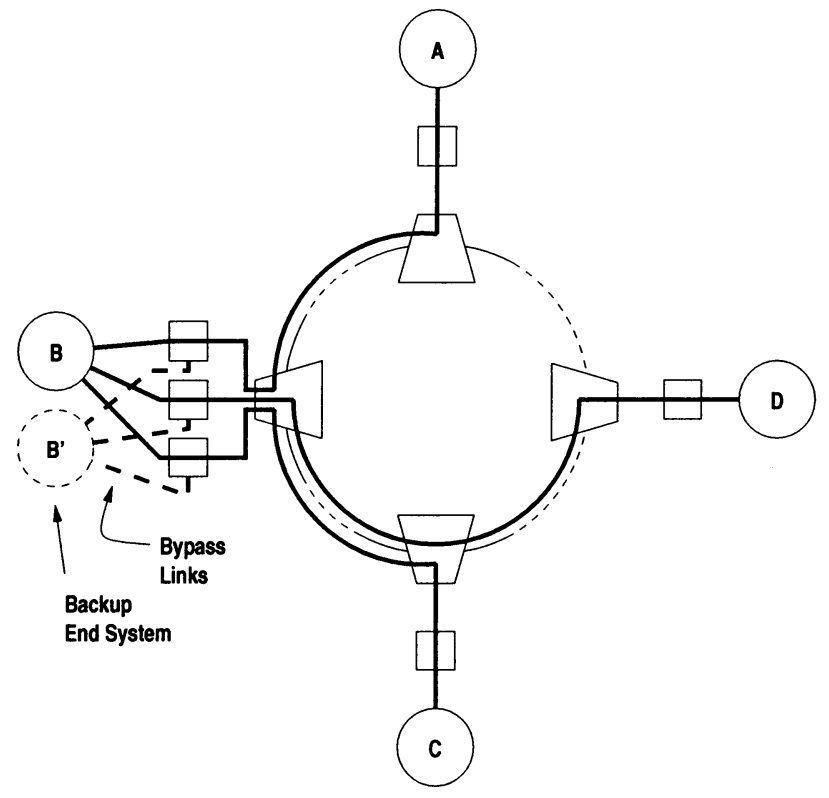

Figure 4: A star network over a WDM ring

\section{ACKNOWLEDGMENTS}

This work was supported by the German Federal Ministry of Education and Research (BMBF) and by Siemens AG, Munich in the KomNet project. The content of this paper is solely the responsibility of the authors. 


\section{References}

[1] D.A. Schupke. Broadband Internet Access using Gigabit Ethernet over Wavelength-Division Multiplexing (WDM) Networks. In Proceedings of the Fifth EUNICE Open European Summer School, Barcelona, Spain, 1999.

[2] M. Jäger, H.-M. Foisel, H.-M. Westphal, J. Chawki, K. Ovsthus, and J.-C. Bischoff. Evaluation of Network Architectures for the Integration of IP over Optical Networks. In 2nd Int. Workshop on the Design of Reliable Communication Networks (DRCN), April 2000.

[3] R. Ramaswami and K.N. Sivarajan. Optical Networks: A Practical Perspective. Morgan Kaufmann Publishers, 1998.

[4] Institute of Electrical and Electronics Engineers (IEEE). 802.3z: Media Access Control (MAC) Parameters, Physical Layer, Repeater and Management Parameters for $1000 \mathrm{Mb} / \mathrm{s}$ Operation, 1998. 